\title{
CHARACTERISTICS OF THE INVOLVEMENT OF THE AMYGDALA IN THE RECOGNITION OF EMOTIONAL EXPRESSIONS: A REVIEW OF NEUROPSYCHOLOGICAL RESEARCH
}

\author{
Wataru SATO $^{1)}$ and Toshiya MURAI ${ }^{1)}$ \\ ${ }^{1)}$ Kyoto University, Japan
}

\begin{abstract}
In this article, we review existing empirical research into the effects of damage to the amygdala on the recognition of facial, bodily and vocal emotional expressions. The evidence indicates that amygdala damage impairs the recognition of emotional expressions. Based on these neuropsychological data, together with anatomical, physiological, neuroimaging and psychological data, we propose that the characteristics of the involvement of the amygdala in the recognition of emotional expressions in normal brain are as follows: (1) The amygdala is involved in the recognition of emotional expressions irrespective of the stimulus type or the sensory modality; (2) The amygdala is necessary not only for the acquisition of emotional knowledge during development but also for online recognition processes in adults; (3) The amygdala is involved particularly in the recognition of negative emotional expressions, although the specific emotional categories in which the amygdala is involved differ among stimuli and subjects, i.e., the amygdala is not necessarily involved only in recognizing fear; (4) The amygdala inhibits the tendency to misrecognize negative emotional expressions as being positive.
\end{abstract}

Key words: emotional expression recognition, amygdala, neuropsychology

Recognizing other individuals' emotional expressions is indispensable for human social interactions. Emotional expressions are displayed through various channels including facial displays, bodily postures, and vocal sounds (Vargas, 1986). The recognition of emotional signals of others allows us to coordinate inter-individual relationship appropriately.

Impairment in the recognition of emotional expressions following amygdala damage, which was reported first by Adolphs, Tranel, Damasio, and Damasio (1994) and has been replicated since in several studies, stands out as one of the most important discoveries in the literature on the recognition of emotion. The amygdala is an almond shaped gray matter positioned in the anteromedial part of the temporal cortex (Fig. 1). In animal studies, it has long been known that bilateral damage to the amygdala causes an abnormal evaluation of environmental stimuli that have emotional content (Amaral et al., 2003).

Correspondence concerning this article should be addressed to Wataru Sato, Department of Human and Environmental Studies, Kyoto University, Yoshida-Nihonmatsu-cho, Sakyo-ku, Kyoto 606-8501, Japan (email: L50158@sakura.kudpc.kyoto-u.ac.jp).

The authors sincerely thank Professor Sakiko Yoshikawa and Tatsuru Kurimoto for their helpful advice. This study was supported by a Research Fellowship of the Japan Society for the Promotion of Science, and was carried out with assistance from the Advanced and Innovational Research program in Life Sciences from the Ministry of Education, Culture, Sports, Science and Technology, the Japanese Government. 
For example, monkeys with bilateral damage to the amygdala readily approach highly aversive stimuli (e.g., snakes) that are avoided by normal monkeys. Whether the amygdala plays a similar role in humans remained unclear for a long period, because clinical observations did not reveal evident changes in behavior following amygdala damage (Halgren, 1992). Recent advances in neuropsychological studies, however, have markedly changed this situation. Close examination in psychological experiments of patients with amygdala damage has revealed that there is impairment of the recognition of emotional expressions of others and clarified the detailed characteristics of this impairment.

In this article, we review the data from neuropsychological studies of the effects of damage to the amygdala on the recognition of emotional expressions. First, we briefly summarize anatomical and physiological information about the amygdala. Second, we review the empirical studies of the recognition of emotional expressions in patients with amygdala damage. Finally, based on the neuropsychological data, together with data from neuroimaging and psychological studies of normal subjects and the results of studies of animals, we propose some of the characteristics of the involvement of the amygdala in the recognition of emotional expressions in the normal brain.

\section{Anatomy And Physiology of the Amygdala}

The amygdala is an almond-shaped mass of gray matter located bilaterally within the anteromedial part of the temporal cortex. The amygdala comprises four major nuclear groups, namely the central nucleus, the medial nucleus, the cortical and basomedial nuclei, and the lateral and basolateral nuclei. Because each of these cell groups has unique pattern of connectivity, it has been argued that the amygdala cannot be regarded as a unified functional component (e.g., Swanson \& Petrovich, 1998). However, there are dense connections among these cell groups, and other studies, including a recent study in which an evolutional approach was used (Barton, Aggleton, \& Grenyer, 2003), have stressed the functional integration of the amygdala.

Anatomical studies in monkeys have provided the information about the connectivity of the primate amygdala. As inputs, the amygdala receives projections from unimodal or polymodal sensory regions representing each of the sensory modalities (Amaral, Price, Pitkanen, \& Carmichael, 1992). This anatomical arrangement suggests that the amygdala may be involved in the processing of sensory stimuli of various types of modality. As regards visual and auditory inputs, the amygdala receives inputs from areas in the superior temporal sulcus associated with unimodal and polymodal sensory processing (Stefanacci \& Amaral, 2000), which have been shown to contain cell populations that respond selectively to conspecific facial, bodily, and vocal signals (Karnath, 2001; Perrett, 1999). This suggests that the amygdala is located in a position that is appropriate for involvement in the processing of facial, bodily, and vocal emotional expressions.

As outputs, the amygdala has dense projections to many brain regions, including

striatum, ventral striatum, hypothalamus, brainstem, and cortical regions (Amaral et al., 
1992), which are important in implementing emotion-related muscular, autonomic, behavioral, and cognitive responses (LeDoux, 1996). Based on these anatomical relationships, some researchers have suggested that the amygdala acts as an interface between sensory stimuli and emotional responses (e.g., Ghashghaei \& Barbas, 2002). Among such connectivity, it is important for the discussion that follows to note that the amygdala sends prominent projections to the nucleus accumbens (Russchen, Bakst, Amaral, \& Price, 1985), which constitutes an essential neural circuit for reward-related behaviors (Blum, Cull, Braverman, \& Comings, 1996).

Physiological studies in monkeys have revealed that some cells in the amygdala are active specifically in response to conspecific facial, bodily, or vocal expressions of emotion (Kling \& Brothers, 1992). Several neuroimaging studies in humans (e.g., Breiter et al., 1996) have revealed that the amygdala is activated in response to others' facial, bodily, and vocal expressions of emotion, particularly of negative emotions, though the findings have been inconsistent with respect to the positive emotions. These data suggest that the amygdala is involved in the processing of various modes of perceived emotional expressions.

\section{EVIDENCE OF IMPAIRED RECOGNITION OF EMOTIONAL EXPRESSIONS AFter Amygdala Damage}

To investigate the performance of emotional expression recognition in amygdaladamaged patients, many studies have been conducted using facial expressions as stimuli. However, stimuli have not been limited to facial displays, but have included bodily postures and vocal sounds. These studies are reviewed below.

\section{Recognition of Emotional Expressions in Facial Displays}

Amygdala damage has been reported to impair the recognition of facial expressions of basic emotions. The research by Adolphs et al. (1994) was the first to report such impairment. These authors examined a 32-year-old woman (SM) with early bilateral damage to the amygdala owing to a genetic disease, namely Urbach-Wiethe disease. Magnetic resonance imaging (MRI) showed nearly complete damage to the bilateral amygdala, and almost no abnormalities in other regions except for minor damage to the entorhinal cortex contiguous with the amygdala damage. SM had no apparent reduction of intelligence, had normal visual discrimination abilities, and had normal abilities to identifying familiar people from faces and discriminating unknown faces. In the experiment, photographs of facial expressions of six basic emotions (anger, disgust, fear, happiness, sadness, and surprise) and neutral emotion selected from the standard facial image set of Ekman and Friesen (1974) were shown to SM. SM was asked to rate the intensity with which each of the six basic emotions was represented in the photographs of stimulus facial expressions. Compared to normal and brain-damaged control subjects, SM showed disproportionately abnormal scores for recognizing facial expressions that represented fear and had a lesser abnormality in recognizing facial expressions of anger 
and surprise. In addition, SM was asked to rate the similarity among the different facial expressions, and she exhibited impaired similarity judgment, compared to brain-damaged controls. SM was also impaired in the drawing of facial expressions of fear from memory (Adolphs, Tranel, Damasio, \& Damasio, 1995).

Such impairment in the recognition of emotional facial expressions after damage to the amygdala was confirmed by subsequent studies of single cases with congenital or acquired damage to the amygdala. Young and his colleagues (Young et al., 1995; Young, Hellawell, van de Wal, \& Johnson, 1996) investigated a 51-year-old woman (DR) who received a series of bilateral amygdalotomies as an adult following intractable seizure activity of bilateral origin. They found that matching and labeling of facial expression of basic emotions was impaired in DR not only for static but also for moving stimuli, and her mental imagery of the facial expressions was impaired, as compared to that of normal controls. Sprengelmeyer et al. (1999) evaluated a 50-year-old man (NM) with bilateral amygdala gliosis, using a labeling task in which prototypical and morphed expressions were presented. They found that recognition of fear by NM was impaired as compared to normal controls. Adolphs, Russell, and Tranel (1999) assessed the rating of facial expressions of basic emotions in the abovementioned bilateral amygdala-damaged patient SM, using two scales of emotion dimension, namely valence and arousal. When compared to normal controls, SM assigned abnormally low arousal scores to all negative facial expressions (fear, anger, disgust, and sadness), particularly to fearful and angry expressions. Anderson and Phelps (2000) investigated a 54-year-old woman (SP), who had bilateral damage to the amygdala due to a right anteromedial temporal lobectomy at 48 years of age in addition to gliosis that extended into the left amygdala (which was observed prior to the right temporal resection). SP was investigated using a rating task for facial expressions of basic emotions. The researchers found that the recognition of fear was severely impaired in SP and in addition found that SP had deficits in the recognition of disgust, sadness, and (to a lesser extent) happiness, relative to normal controls.

Impaired recognition of facial expressions has also been confirmed in studies of multiple cases with congenital or acquired damage to the amygdala. Calder et al. (1996) evaluated two patients with bilateral amygdala damage (including abovementioned patient DR) in a labeling task using continua of morphed facial expressions, i.e., intermediate expressions obtained by combining two prototypical expressions. Recognition of fearful and angry expressions was impaired in both patients as compared to normal controls. Broks et al. (1998) evaluated facial expression labeling in three bilateral and one unilateral (left-sided) amygdala-damaged patients. Each of these patients performed worse than normal controls in the recognition of fearful expressions. Adolphs, Tranel, et al. (1999) tested nine patients with bilateral damage to the amygdala using a rating task and facial expressions of basic emotions. The patients exhibited impaired recognition of facial expressions of all emotions except for happiness as compared to normal and braindamaged controls; impairment was most evident for fear and anger. Rapcsak et al. (2000) assessed the labeling of facial expressions of basic emotions in two bilateral and 11 unilateral (eight right-sided, three left-sided) amygdala-damaged patients. They found that the recognition of fear and disgust by these patients was impaired as compared to 
normal controls, although there was no difference in the performance of these patients relative to brain-damaged controls. Anderson, Spencer, Fulbright, and Phelps (2000) investigated 23 patients with unilateral damage to the amygdala (12 left-sided, 11 rightsided) using a rating task and facial expressions of basic emotions. Patients with damage to the right amygdala exhibited impaired recognition of facial expressions of some emotions, clearly for sadness, disgust, and happiness (although the impairment of recognition of happiness was the less evident than other two emotions). Adolphs, Tranel, and Damasio (2001) tested 26 unilateral amygdala-damaged patients (15 left-sided, 11 right-sided) using a rating task and facial expressions of basic emotions, and observed impaired recognition of fearful expressions in the right amygdala-damaged patients, as compared to brain-damaged controls. Brierley, Medford, Shaw, and David (2004) investigated three bilateral and 25 unilateral (11 left-sided, 14 right-sided) amygdaladamaged patients using a labeling task and morphed facial expressions. Two of the three bilateral amygdala-damaged patients were consistently deficient in recognizing angry and disgusted facial expressions, as compared to normal controls. Unilateral amygdaladamaged patients (irrespective of which hemisphere was damaged) were deficient in recognizing anger, disgust, and fear, as compared to normal controls.

Some studies have investigated qualitative changes in the recognition of emotional facial expression in amygdala-damaged patients, and have reported that there is an intriguing bias towards recognizing negative expressions as being positive. Sato et al. (2002) evaluated the impaired recognition of facial expressions of basic emotions in a 37-year-old woman (HY) with bilateral damage to the amygdala owing to Herpes simplex-induced encephalitis at 27 years of age. An MRI image of this patient's brain is presented in Figure 1: bilateral focal brain damage within the amygdala and the adjacent areas is evident. HY had no problems in the recognition of familiar faces and discrimination of unfamiliar faces, and showed no marked decrease in intelligence. In Experiment 1, a labeling task was conducted in which prototypical facial expressions were presented. The pattern of errors made by HY in recognizing fearful or angry facial expressions was distinct from those of normal and brain-damaged controls and indicated that HY confused fear and anger with happiness. In Experiment 2, HY was presented with morphed facial expressions that blended expressions of happiness and fear, happiness and anger, or happiness and sadness (Fig. 2), and was requested to categorize the expressions by means of a two-way forced choice selection. HY categorized the morphed expressions of fear and anger that were blended with some happy content as being happy facial expressions more frequently than did normal controls (Fig. 3). These results indicate that amygdala damage elicited a bias towards positive evaluations of fearful or angry facial expressions. A similar positive bias was described by Adolphs and Tranel (2003) for a study of the recognition of basic emotions from social scenes that either did or did not include facial expressions. Four bilateral and 23 unilateral (10 left-sided, 13 right-sided) amygdala-damaged patients were studied. Compared to normal and brain-damaged controls, the bilateral amygdala-damaged patients were impaired in recognizing scenes with angry facial expressions, and these patients frequently mistook angry facial expressions for happy expressions; none of the control subjects made such mistakes. 


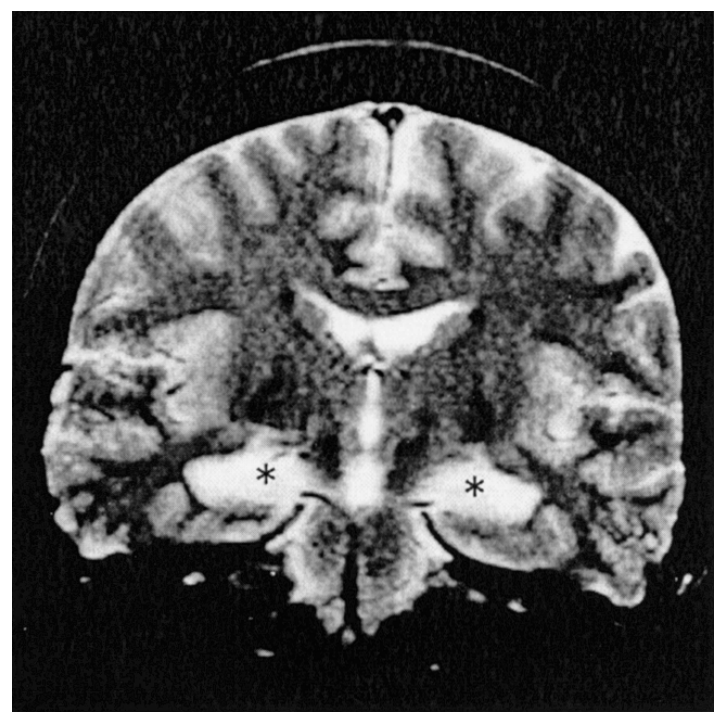

Fig. 1. An example of T2-weighted magnetic resonance imaging (MRI) of the brain of a patient with bilateral damage to the amygdala (Sato et al., 2002). The location of the amygdala is indicated by asterisks. Note that bilateral focal brain damage is evident in the amygdala and adjacent areas.

Adolphs and Tranel (2004) investigated the rating and labeling of morphed facial expressions that blended happiness and sadness in five bilateral and 27 unilateral (16 leftsided, 11 right-sided) amygdala-damaged patients. The bilateral amygdala-damaged patients exhibited impaired recognition of sad expressions, as compared to brain-damaged controls, and their rating revealed the intrusion of disgust and happiness into the recognition of sad expressions.

In the literature, some null results have been reported with regards to the recognition of facial expressions of emotions. Hamann et al. (1996) investigated ratings of facial expressions of basic emotions in two bilateral amygdala-damaged patients using the same materials and procedure as those used by Adolphs et al. (1994). They found no impairment in the patients as compared to normal controls. Hamann and Adolphs (1999) also reported no abnormalities in the judgment of the similarity of emotional expressions in these patients, though one of the patients did exhibit a slight deficit in the ratings of happy and fearful expressions as compared to brain-damaged controls. However, in a subsequent study by Schmolck and Squire (2001) in which these patients and one additional bilateral amygdala-damaged patient were investigated in detail using labeling tasks and facial expressions of basic emotions, there was consistent impairment of the recognition of fearful expressions as compared to normal controls.

In contrast to all of the abovementioned studies that focused on facial expressions of basic emotions, Adolphs, Baron-Cohen, and Tranel (2002) examined the recognition not only of basic emotions but also of social emotions from the eye region (i.e., eyes and brows), applying an established test set (Baron-Cohen, Wheelwright, Hill, Raste, \& Plumb, 2001). The defined social emotions included guilt, arrogance, admiration, and 

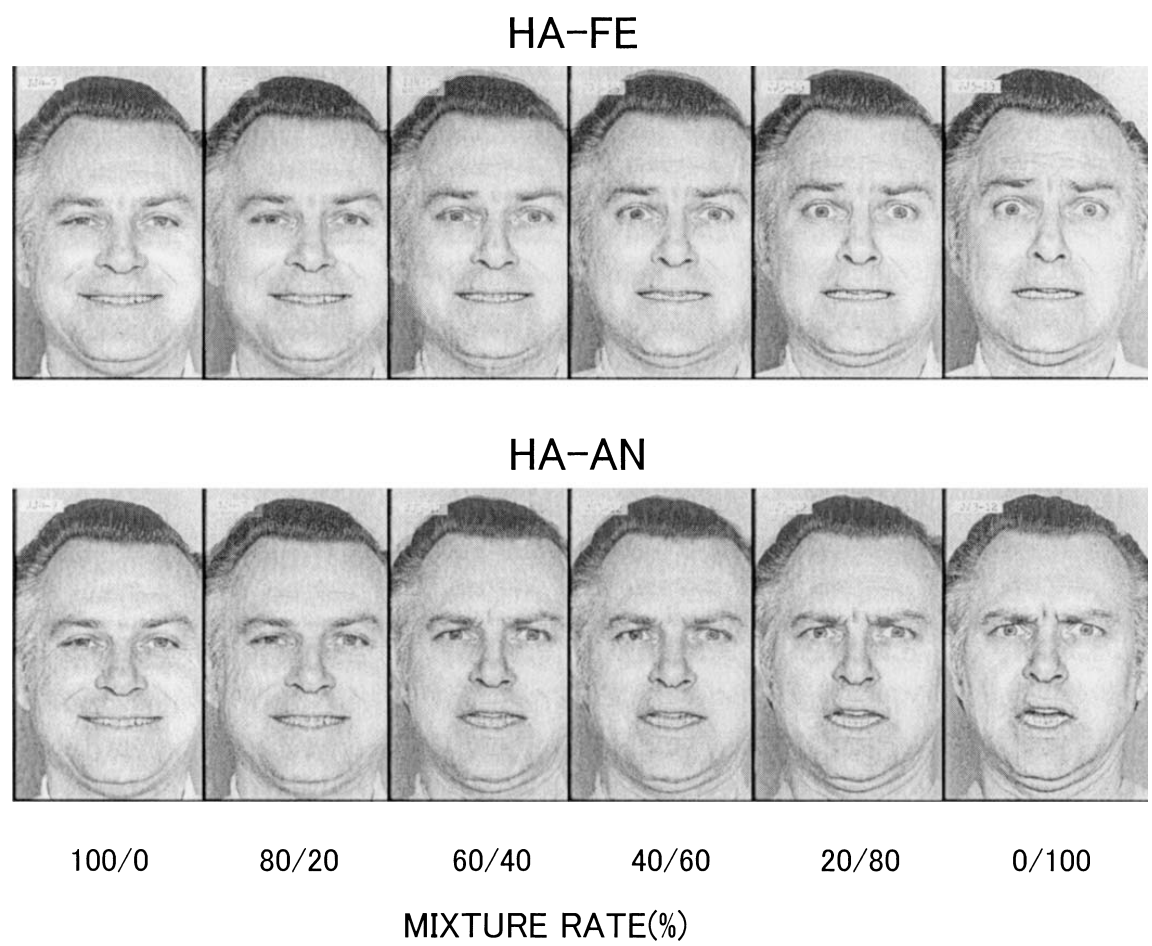

Fig. 2. Examples of morphed facial expressions used as stimuli by Sato et al. (2002). Upper and lower lines indicate the interpolated continuums of happiness-fear (HA-FE) and happiness-anger (HA$\mathrm{AN}$ ), respectively. From left to right, each face blends the partner emotion of happiness (i.e., fear, anger, or sadness) at rates of $0,20,40,60,80$, and $100 \%$, respectively.

flirtatiousness. They investigated two bilateral and 30 unilateral (16 left-sided, 14 rightsided) amygdala-damaged patients. Compared to brain-damaged controls, bilateral and unilateral amygdala-damaged patients exhibited clear impairment of the recognition of the social emotions. Similarly, Stone, Baron-Cohen, Calder, Keane, and Young (2003) investigated the recognition of mental states that included complex emotions (e.g., sympathetic) from the eye region using a test set of Baron-Cohen, Wheelwright, and Jolliffe (1997). They investigated two patients with bilateral damage to the amygdala, and found that both patients were impaired as compared to normal controls.

\section{Recognition of Emotional Expressions in Bodily Postures}

Amygdala damage has been reported by Sprengelmeyer et al. (1999) to cause impairment of the recognition of emotional expressions in bodily postures. In their study, the abovementioned bilateral amygdala-damaged patient $\mathrm{NM}$ was presented with photographs of bodily postures in which actors expressed basic emotions (their faces were masked), and was asked to select appropriate emotional labels for the photographs. The recognition of fear by NM was poorer than normal controls. 

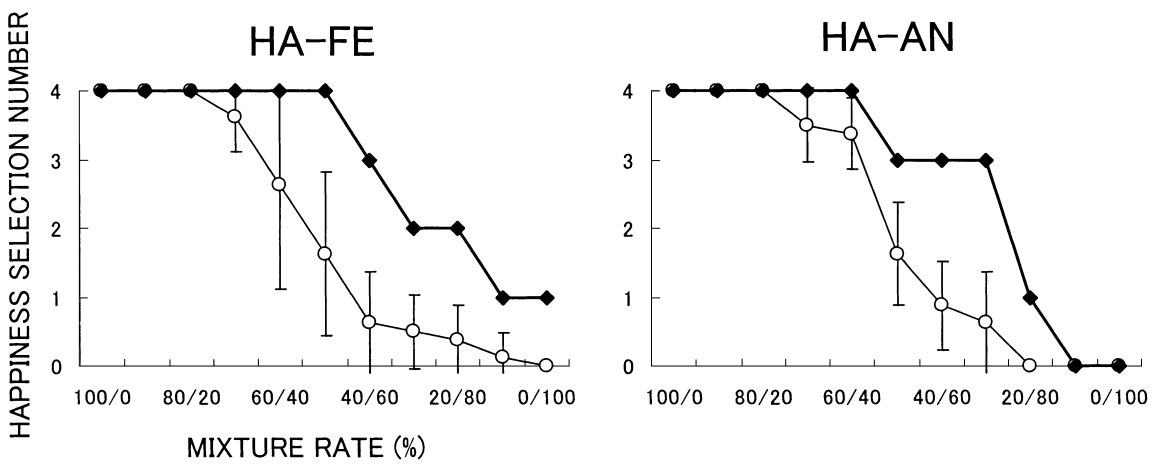

Fig. 3. Results of Sato et al. (2002). Happiness ratings in the recognition of emotion in morphed facial expressions by normal controls (NORMAL) and by a patient with damage to the amygdala (AMYG DAMAGE) are presented. The figures to the right and left indicate the results of responses to the morphed continuums happiness-fear (HA-FE) and happiness-anger (HA-AN), respectively. Note that the patient with damage to the amygdala categorized the morphed fearful and angry expressions blended with some happy content as happy facial expressions more frequently than did the normal controls.

\section{Recognition of Emotional Expressions in Vocal Sounds}

Amygdala damage has been reported to cause impairment of the recognition of emotion represented by auditory emotional expressions. Scott et al. (1997) asked the abovementioned bilateral amygdala-damaged patient DR to listen to and assign emotional labels to neutral content sentences and words intoned with emotions as well as non-verbal sounds with emotions (e.g., growling for anger). DR consistently exhibited relatively poor recognition of fear and anger as compared to normal controls. Anderson and Phelps (1998) investigated the abovementioned bilateral amygdala-damaged patient SP in a labeling and rating task and neutral words and nonverbal sounds that were intoned with emotion. SP consistently exhibited impaired recognition of disgust in both types of stimulus as compared to normal controls. Sprengelmeyer et al. (1999) evaluated the recognition of emotion in emotional nonverbal sounds in the abovementioned bilateral amygdala-damaged patient NM, and found impaired recognition of fear in this patient as compared to normal controls. Adolphs and Tranel (1999b) testd two bilateral and seven unilateral (two left-sided, five right-sided) amygdala-damaged patients using a rating task and neutral sentences spoken with specific emotional tones. One of the bilateral amygdala-damaged patient exhibited impaired recognition of anger, and one unilateral amygdala-damaged patient exhibited impaired recognition of fear, as compared to braindamaged controls. The bilateral amygdala-damaged patient was impaired in recognizing fear (at the maximal rating); Intriguingly, this patient misrecognized fear as surprise or happiness. Adolphs et al. (2001) tested 26 patients with unilateral damage to the amygdala (15 left-sided, 11 right-sided) using a prosody recognition task. Patients with 
either left- or right-sided amygdala-damaged patients performed substantially worse than normal controls in recognizing fear, although a similar difficulty in recognizing fear was also exhibited by brain-damaged controls. Brierley et al. (2004) assessed three bilateral and 25 unilateral (11 left-sided, 14 right-sided) amygdala-damaged patients using a labeling task and neutral-content words that were intoned with emotions. Two of the three bilateral amygdala-damaged patients consistently exhibited a deficit in the recognition of fearful vocal expressions as compared to normal controls. Unilateral amygdala-damaged patients were deficient in recognizing happiness as compared to normal controls.

\section{CHARACTERISTICS OF THE INVOLVEMENT OF THE AMYGDALA IN THE RECOGNITION OF EMOTIONAL EXPRESSIONS}

As reviewed in the section above, there is ample evidence that the recognition of emotional expressions is impaired following damage to the amygdala. Such impairment has been present even when the intelligence and basic face perception ability of patients is normal (e.g., Sato et al., 2002), which suggests that impairment of the recognition of emotional expressions cannot be attributed to these factors. Impairment of emotional expression recognition has been demonstrated in some cases in which the lesion is restricted almost exclusively to the amygdala (e.g., Sato et al., 2002), which suggests that such impairment cannot be attributed exclusively to damage in other brain regions. Because various experimental paradigms have been used in different studies, the impairment of the recognition of emotional expressions cannot be explained by certain experimental artifacts.

The experimental evidence that has been gathered to date from studies of patients with damage to the amygdala provides clues to the characteristics of the involvement of the amygdala in the recognition of emotional expressions in the normal brain. Based on these studies and other anatomical, physiological, neuroimaging and psychological data, we propose that the major characteristics of the involvement of the amygdala in the recognition of emotional expressions are as follows.

(1) The amygdala is involved in the recognition of emotional expressions irrespective of the stimulus type or the sensory modality.

Impairment of the recognition of emotional expressions following amygdala damage has been reported based on experiments in which multiple types of visual expression have been used, including displays of faces (e.g., Adolphs et al., 1994) and bodily postures (Sprengelmeyer et al., 1999). Impairment of emotional expression recognition has also been shown based on experiments in which auditory representations of emotional expressions were used (e.g., Scott et al., 1997). Therefore, it would appear that the processing of emotions by the amygdala is not specific to the stimulus type or the sensory modality; rather, the amygdala would appear to process the recognition of emotions nonspecifically (i.e., generally).

Our proposal that the amygdala is not specific to certain types of stimulus is 
consistent with neurophysiological studies in monkeys (e.g., Ono \& Nishijo, 1999) and cats (e.g., Maeda, Morimoto, \& Yanagimoto, 1993), which have revealed that the neuronal activity in the amygdala elicited by visual stimuli does not reflect the physical features of the stimuli, but is related to the emotional content of the stimuli. Neuroimaging studies in humans have also shown that the amygdala is active in response not only to facial but also to bodily emotional expressions (e.g., Hadjikhani \& de Gelder, 2003).

Consistent with our proposal that the amygdala is involved in the multimodal recognition of emotional expressions, anatomical studies in monkeys revealed that the amygdala receives convergent projections from the sensory areas of different modalities (Amaral et al., 1992). Lesion studies in rats indicated that the amygdala is involved in the processing of the emotional content of both visual and auditory sensory stimuli (Gallagher \& Chiba, 1996). Human neuroimaging studies have also shown that the amygdala is active not only to facial but also to vocal emotional expressions (Phillips, Young, et al., 1998).

It should be noted that some studies have reported a differential pattern of impairment of recognition of facial and vocal representations of emotion (e.g., Anderson \& Phelps, 1998), although the pattern of impairment of recognition of facial and vocal expressions of emotion was consistent in other studies (e.g., Sprengelmeyer et al., 1999). Therefore, we think that there are idiosyncratic differences in the involvement of the amygdala in recognizing emotional expressions among different types of stimuli (see below).

(2) The amygdala is necessary not only for the acquisition of emotional knowledge during development but also for online recognition processes in adults.

Amygdala damage has been shown to cause impairment of emotional expression recognition regardless of whether the damage occurred at an early stage of development owing to congenital disorder (e.g., Adolphs et al., 1994) or in adulthood (e.g., Young et al., 1995). In the early literatures, one study of a patient with congenital damage to the amygdala (Adolphs et al., 1994) reported impaired recognition of emotional facial expressions, while another study of patients with damage to the amygdala that was acquired in adulthood did not find any such impairment (Hamann et al., 1996). Based on these findings, some researchers (e.g., Hamann et al., 1996) proposed the possibility that impairment of emotional expression recognition may develop only when the amygdala damage occurs during an early stage of development. The researchers suggested that the amygdala is involved in the formation of emotional knowledge, which is retained in different area of the brain, and that the amygdala is not involved in the online recognition of emotional expressions in adults. This is analogous to the role of the hippocampus in memory. However, this proposal is no longer supported: data from neuropsychological studies reviewed here indicate that the amygdala plays an important role in the process of online recognition of emotional expressions in adults.

The idea that the amygdala is involved in the online processing of emotional expressions in adults is consistent with the results of several neuroimaging studies, in 
which the amygdala was activated in adults in response to the presentation of emotional expressions (e.g., Breiter et al., 1996).

(3) The amygdala is involved particularly in the recognition of negative emotional expressions, although the specific emotional categories in which the amygdala is involved differ among stimuli and subjects, i.e., the amygdala is not necessarily involved only in recognizing fear.

Many studies have shown that damage to the amygdala impairs the recognition not of overall emotions but of specific emotional categories. Most studies have reported severe impairment of the recognition of fearful expressions (e.g., Adolphs et al., 1994), and the next most frequently reported impairment is the recognition of angry expressions (e.g., Calder et al., 1996). Some studies have reported severe impairment of the recognition of disgust (e.g., Anderson \& Phelps, 1998) and sadness (e.g., Anderson et al., 2000). Compared to such negative emotions, the impairment of the recognition of happiness and surprise has been reported in relatively few studies, and in almost all such studies, researchers have noted that the impairment of these emotions as less evident than the impairment of other negative emotions (e.g., Anderson \& Phelps, 2000). Based on these findings, we propose that the amygdala is involved particularly in the recognition of negative emotional expressions. It does not appear that the amygdala is specifically involved in the recognition of fearful expressions, which has been previously proposed by some researchers (e.g., Adolphs et al., 1995).

The emotional categories that were impaired following damage to the amygdala are not consistent among different studies. This indicates that the effect of the amygdala damage on the recognition of emotional expressions differs among individuals. In addition, some studies (e.g., Anderson \& Phelps, 1998) have reported that the patterns of impairment of different emotional categories differed for recognition by a single patient of facial and vocal expressions. Together, it appears that the emotional categories that the amygdala is involved in recognition process vary among different subjects and stimuli.

Regarding the valence and/or categories of emotion in which the amygdala is involved, data from animal studies are inconsistent with the neuropsychological data that we have reviewed. Several physiological studies in rats, cats, and monkeys (e.g., Ono \& Nishijo, 1999) showed that the amygdala is involved in processing both negative and positive emotional stimuli. Similarly, the results of neuroimaging studies do not provide a conclusive answer: although activation of the amygdala in response to negative expressions has been frequently reported (e.g., Breiter et al., 1996), some studies have reported activation of the amygdala in response to happy (e.g., Breiter et al., 1996) and surprised (e.g., Kim, Somerville, Johnstone, Alexander, \& Whalen, 2003) expressions. There is also inconsistency with regards to the effect of specific negative emotional categories. For example, Blair, Morris, Frith, Perrett, and Dolan (1999) reported increased activity in the amygdala in response to sad facial expressions, whereas Phillips, Bullmore, et al. (1998) did not. Clearly, this issue requires further investigation.

Another important issue is the effect of amygdala damage on the recognition of social and complex emotions (e.g., guilt) in faces, which has been shown in some recent 
studies to be impaired (e.g., Adolphs et al., 2002). More work is required to investigate in detail the pattern of impairment in the recognition of social and complex emotions in patients with amygdala damage.

\section{(4) The amygdala inhibits the tendency to misrecognize negative emotional expressions as being positive.}

Some studies have shown that negative emotional expressions in other individuals are misrecognized as happiness by patients with damage to the amygdala (e.g., Sato et al., 2002). These observations were made in studies in which various different experimental paradigms were used, including the presentation of facial (e.g., Sato et al., 2002) and vocal (e.g., Adolphs \& Tranel, 1999b) expressions as stimuli. This positive recognition bias for negative emotional expressions is intriguing, because it is very rare in normal subjects (Sato et al., 2002). The available evidence suggests the possibility that the amygdala inhibits the tendency to misrecognize negative emotional expressions as being positive.

Regarding this idea, some studies have reported a similar positive bias in the evaluation of emotionally negative stimuli other than basic emotions in patients with damage to the amygdala. For example, Adolphs, Tranel, and Damasio (1998) asked three bilateral amygdala-damaged patients to rate the trustworthiness and approachability of various faces. Using these scores, the 50 stimuli that were considered to be the most negative and the 50 stimuli that were considered to be the most positive by normal controls were analyzed. The patients with bilateral amygdala damage tended to rate negative faces as positive. Similar results were confirmed by using a task in which patients were required to select more trustworthy or approachable face from a pair of faces. Using the emotional rating of non-social stimuli (e.g., paintings of landscape), the pleasantness of which the subjects were asked to assess, Adolphs and Tranel (1999a) investigated two patients with bilateral amygdala damage. They found that stimuli that were considered by normal subjects to be the most negative were regarded consistently as positive by both patients with bilateral damage to the amygdala.

A positive bias in the recognition of negative emotional situations and social behaviors in amygdala-damaged patients has also been reported anecdotally. Broks et al. (1998) depicted an exampler of positive recognition error in a bilateral amygdaladamaged patient. When the patient and her husband were returning to their hotel after an evening out on holiday, they were mugged by some youths. A physical struggle between her husband and the youths ensued, along with a loud aggressive verbal exchange. Regarding this struggle, the patient thought that they were merely larking around. Damasio (1999) also described a positive social behavior bias in the aforementioned bilateral amygdala-damaged patient, SM. He noted that SM excessively and inappropriately approached people and situations with a predominantly positive attitude. For example, SM hugged and touched unfamiliar individuals shortly after being introduced to them.

The quantitative changes in emotional evaluations and behaviors that follow damage to the amygdala in humans appear to be analogous to the changes that occur in monkeys. Emery et al. (2001) investigated the effect of amygdala damage in dyadic interactions with 
unfamiliar conspecific animals; normally, monkeys in this situation exhibit wariness that is often accompanied by aggression. The focal bilateral amygdala damage was prepared by the stereotaxic ibotenic acid injection into the amygdala, which destroyed the amygdala cell bodies, minimizing the damage to adjacent areas and leaving fibers of passage through the amygdala intact. Through three experiments, they consistently found that monkeys with damage to the amygdala were rarely tense during interactions with unfamiliar conspecific animals and that these animals frequently exhibited positive social behavior (i.e., affiliative behavior such as cooing vocalizations) that was directed towards the unfamiliar conspecifics.

Studies in animals may provide further information about the neural circuits that underlie the positive recognition/behavior bias towards negative emotional stimuli after amygdala damage. As mentioned in Section 1, anatomical studies in monkeys have shown that the amygdala sends dense projections to the nucleus accumbens (Russchen et al., 1985). Anatomical studies in rats have shown that both the amygdala and nucleus accumbens receive dopamine (DA) projections that originate within the ventral tegmental area of the brain-stem (Callahan, de la Garza, \& Cunningham, 1997). DA activity within the nucleus accumbens has been shown to be important in reward-related activity in rats and monkeys (Joseph, Datla, \& Young, 2003), and this has been proposed also to be the case in humans (Blum et al., 1996). In addition, DA neurons in the nucleus accumbens have been shown to be active in rats in response to negative emotional stimuli (Giorgi, Lecca, Piras, Driscoll, \& Corda, 2003; Stevenson \& Gratton, 2003), although the specific function of this neuronal activity remains unclear. Intriguingly, previous in vivo (Louilot, Simon, Taghzouti, \& Le Moal, 1985; Hurd, McGregor, \& Ponten, 1997; Stevenson \& Gratton, 2003, in press; Stevenson, Sullivan, \& Gratton, 2003) and in vitro (Simon et al., 1988) studies in rats have revealed that lesion of the DA neurons within the amygdala induce enhanced DA activity in the nucleus accumbens. Based on these data, we speculate that some psychological process related to positive emotion may be implemented by DA activity within the nucleus accumbens in response to negative emotional stimuli, and that one of the amygdala functions may be to inhibit these functions of the nucleus accumbens functions appropriately.

Previous psychological studies of emotional experience have indicated that humans could simultaneously experience fear and other positive emotions, such as interest and enjoyment (Philippot, 1993; Gross \& Levenson, 1995; Niedenthal, Halberstadt, \& InnesKer, 1999). For example, Gross and Levenson (1995) selected two films that would best induce fear from among more than 250 films and presented these films to subjects. The subjects reported experiencing both fear and interest while viewing the two films. Ekman and Friesen (1975) pointed out that some people (e.g., soldiers and mountain climbers) can enjoy fearful experience and that these people could be termed brave, courageous or daring. To be brave by the simultaneous occurrence of negative and positive emotional experience would be biologically adaptive in some situations. Similarly, if negative emotional stimuli were to evoke a positive emotion, such as interest or a challenge, together with the simple recognition that the stimuli were fearful or dangerous, this would allow an individual to explore or interact with such stimuli. At the same time, it is obvious 
that overly optimistic recognition of negative stimuli as positive would threaten the safety of an individual, which suggests that the tendency to recognize negative stimuli as positive would normally be inhibited. The amygdala may be a neural substrate that is particularly crucial to this inhibitory function.

It is intriguing that the amygdala may inhibit the tendency to misrecognize negative emotional expressions as positive in the normal brain. However, the evidence that is available at present is insufficient to support this proposal conclusively, and further studies are required in this regard.

\section{CONCLUSIONS AND FUTURE DIRECTIONS}

We have reviewed the results of experimental research into the effects of amygdala damage on the recognition of emotional expressions. The data clearly suggests that amygdala damage impairs the recognition of emotional expressions. Based on these data, together with the data from other literatures, we proposed some characteristics of the involvement of the amygdala in the recognition of emotional expressions in the normal brain.

Nevertheless, much remains to be clarified. Most importantly, the precise psychological function of the amygdala in emotional expression recognition remains unclear. A recent functional MRI study by Sato, Yoshikawa, Kochiyama, and Matsumura (2004) provided some clues in this issue. In this study, subjects viewed the angry or neutral expressions of faces that were looking either toward or away from the subjects, and the brain activity of the subjects was measured while they were viewed the faces. Subsequently, the subjects were presented with the same stimuli, and were required to rate the intensity of experienced emotion (i.e., the emotion subjects felt when perceiving the stimulus models' expression) as well as recognized emotion (i.e., the emotion that subjects recognized from the stimulus models' expression) using a 5-point scale of negativity. Regression analyses revealed that the relationship between amygdala activity and experienced emotion was significant and positive, whereas the relationship between amygdala activity and recognized emotion was not significant. These data suggest that amygdala activity in response to the emotional expressions of others may be related to the elicitation of the perceiver's own emotions, but is not related directly to the recognition of facial expressions.

Then, why the amygdala is involved in the recognition of emotional expressions in other individuals? We hypothesize that perceivers refer to their own emotional responses to recognize the emotional states of others. That is, the perception of the emotional expressions of others (e.g., angry expressions) would elicit emotions (e.g., fear) in the perceiver; these emotional reactions would then be utilized as the information by the perceiver to recognize emotional expressions. Previous psychological studies have shown that the emotional expressions of others elicit various types of emotional responses, including subjective, physiological, muscular, and cognitive responses (Frijda, 1986). Because the amygdala sends dense projections to many brain regions (as mentioned 
above), the amygdala would be an appropriate neural substrate with which to implement such widespread emotional reactions. Because the emotional stimuli elicit responses in the amygdala even if the perceivers is not aware of the stimulus (Kubota et al., 2000; Whalen et al., 1998), the rapid and automatic emotional response could affect subsequent intentional judgements. We speculate that perceivers can accomplish the recognition of emotional expressions by intuitively referring to these emotional reactions. We think that this process would be one aspect of a more generalized process of judgment and decisionmaking in humans, which is called heuristic (c.f., Kahneman, 2002); therefore, we shall refer to this process as the 'own emotion heuristic' model of the emotional expression recognition. In summary, we propose that the amygdala plays an important role in rapidly and automatically eliciting a perceiver's own emotional reactions to the emotional expressions of others, and that the emotions elicited in the perceiver are utilized subsequently as information for the heuristic recognition of others' emotions. In future studies, we shall expand upon this theory in detail and aim to provide empirical support for the psychological and mechanisms that are involved.

\section{REFERENCES}

Adolphs, R., Baron-Cohen, S., \& Tranel, D. 2002. Impaired recognition of social emotions following amygdala damage. Journal of Cognitive Neuroscience, 14, 1264-1274.

Adolphs, R., Russell, J. A., \& Tranel, D. 1999. A role for the human amygdala in recognizing emotional arousal from unpleasant stimuli. Psychological Science, 10, 167-171.

Adolphs, R., \& Tranel, D. 1999a. Preferences for visual stimuli following amygdala damage. Journal of Cognitive Neuroscience, 11, 610-616.

Adolphs, R., \& Tranel, D. 1999b. Intact recognition of emotional prosody following amygdala damage. Neuropsychologia, 37, 1285-1292.

Adolphs, R., \& Tranel, D. 2003. Amygdala damage impairs emotion recognition from scenes only when they contain facial expressions. Neuropsychologia, 41, 1281-1289.

Adolphs, R., \& Tranel, D. 2004. Impaired judgments of sadness but not happiness following bilateral amygdala damage. Journal of Cognitive Neuroscience, 16, 453-462.

Adolphs, R., Tranel, D., \& Damasio, A. R. 1998. The human amygdala in social judgment. Nature, 393, $470-474$.

Adolphs, R., Tranel, D., \& Damasio, H. 2001. Emotion recognition from faces and prosody following temporal lobectomy. Neuropsychology, 15, 396-404.

Adolphs, R., Tranel, D., Damasio, H., \& Damasio, A. 1994. Impaired recognition of emotion in facial expressions following bilateral damage to the human amygdala. Nature, 372, 669-672.

Adolphs, R., Tranel, D., Damasio, H., \& Damasio, A. R. 1995. Fear and the human amygdala. The Journal of Neuroscience, 15, 5879-5891.

Adolphs, R., Tranel, D., Hamann, S., Young, A. W., Calder, A. J., Phelps, E. A., et al. 1999. Recognition of facial emotion in nine individuals with bilateral amygdala damage. Neuropsychologia, 37, 11111117.

Amaral, D. G., Bauman, M. D., Capitanio, J. P., Lavenex, P., Mason, W. A., Mauldin-Jourdain, M. L., et al. 2003. The amygdala: is it an essential component of the neural network for social cognition? Neuropsychologia, 41, 517-522.

Amaral, D. G., Price, J. L., Pitkanen, A., \& Carmichael, S. T. 1992. Anatomical organization of the primate amygdaloid complex. In J. P. Aggleton (Ed.), The amygdala: Neurobiological aspects of emotion, memory, and mental dysfunction (pp. 1-66). New York: Wiley-Liss.

Anderson, A. K., \& Phelps, E. A. 1998. Intact recognition of vocal expressions of fear following bilateral 
lesions of the human amygdala. Neuroreport, 9, 3607-3613.

Anderson, A. K., \& Phelps, E. A. 2000. Expression without recognition: Contributions of the human amygdala to emotional communication. Psychological Science, 11, 106-111.

Anderson, A. K., Spencer, D. D., Fulbright, R. K., \& Phelps, E. A. 2000. Contribution of the anteromedial temporal lobes to the evaluation of facial emotion. Neuropsychology, 14, 526-536.

Baron-Cohen, S., Wheelwright, S., Hill, J., Raste, Y., \& Plumb, I. 2001. The "Reading the Mind in the Eyes" Test revised version: a study with normal adults, and adults with Asperger syndrome or highfunctioning autism. Journal of Child Psychology and Psychiatry and Allied Disciplines, 42, 241-251.

Baron-Cohen, S., Wheelwright, S., \& Jolliffe, T. 1997. Is there a "language of the eyes"? evidence from normal adults, and adults with autism or asperger syndrome. Visual Cognition, 4, 311-331.

Barton, R. A., Aggleton, J. P., \& Grenyer, R. 2003. Evolutionary coherence of the mammalian amygdala. Proceedings of the Royal Society of London Series B: Biological Sciences, 270, 539-543.

Blair, R. J., Morris, J. S., Frith, C. D., Perrett, D. I., \& Dolan, R. J. 1999. Dissociable neural responses to facial expressions of sadness and anger. Brain, 122, 883-893.

Blum, K., Cull, J. G., Braverman, E. R., \& Comings, D. E. 1996. Reward deficiency syndrome. American Scientist, 84, 132-146.

Breiter, H. C., Etcoff, N. L., Whalen, P. J., Kennedy, W. A., Rauch, S. L., Buckner, R. L., et al. 1996. Response and habituation of the human amygdala during visual processing of facial expression. Neuron, 17, 875-887.

Brierley, B., Medford, N., Shaw, P., \& David, A. S. 2004. Emotional memory and perception in temporal lobectomy patients with amygdala damage. Journal of Neurology, Neurosurgery and Psychiatry, 75, 593-599.

Broks, P., Young, A. W., Maratos, E. J., Coffey, P. J., Calder, A. J., Isaac, C. L., et al. 1998. Face processing impairments after encephalitis: Amygdala damage and recognition of fear. Neuropsychologia, 36, 5970.

Calder, A. J., Young, A. W., Rowland, D., Perrett, D. I., Hodges, J. R., \& Etcoff, N. L. 1996. Facial emotion recognition after bilateral amygdala damage: Differentially severe impairment of fear. Cognitive Neuropsychology, 13, 699-745.

Callahan, P. M., de la Garza, R., \& Cunningham, K. A. 1997. Mediation of the discriminative stimulus properties of cocaine by mesocorticolimbic dopamine systems. Pharmacology, Biochemistry and Behavior, 57, 601-607.

Damasio, A. R. 1999. The feeling ofwhat happens: Body and emotion in the making of consciousness. New York: Harcourt Brace.

Ekman, P. , \& Friesen, W. V. 1974. Pictures of fcial affect. Palo Alto: Consulting Psychologist.

Ekman, P. , \& Friesen, W. V. 1975. Unmasking the face: A guide to recognizing emotions from facial clues. Englewood Cliffs, NJ: Prentice-Hall.

Emery, N. J., Capitanio, J. P., Mason, W. A., Machado, C. J., Mendoza, S. P., \& Amaral, D. G. 2001. The effects of bilateral lesions of the amygdala on dyadic social interactions in rhesus monkeys (Macaca mulatta). Behavioral Neuroscience, 115, 515-544.

Frijda, N. H. 1986. Facial expression processing. In H. Ellis, M. A. Jeeves, F. Newcombe, \& A. Young (Eds.), Aspects of face processing (pp. 319-325). Dordrecht, The Netherlands: Martinus Nijhoff.

Gallagher, M., \& Chiba, A. A. 1996. The amygdala and emotion. Current Opinion in Neurobiology, 6, 221227.

Ghashghaei, H. T., \& Barbas, H. 2002. Pathways for emotion: interactions of prefrontal and anterior temporal pathways in the amygdala of the rhesus monkey. Neuroscience, 115, 1261-1279.

Giorgi, O., Lecca, D., Piras, G., Driscoll, P., \& Corda, M. G. 2003. Dissociation between mesocortical dopamine release and fear-related behaviours in two psychogenetically selected lines of rats that differ in coping strategies to aversive conditions. European Journal of Neuroscience, 17, 2716-2726.

Gross, J. J., \& Levenson, R. W. 1995. Emotion elicitation using films. Cognition and Emotion, 9, 87-108.

Hadjikhani, N., \& de Gelder, B. 2003. Seeing fearful body expressions activates the fusiform cortex and amygdala. Current Biology, 13, 2201-2205.

Halgren, E. 1992. Emotional neurophysiology of the amygdala within the context of human cognition. In J. P. Aggleton (Ed.), The amygdala: Neurobiological aspects of emotion, memory, and mental dysfunction (pp. 191-228). New York: Wiley-Liss. 
Hamann, S. B., \& Adolphs, R. 1999. Normal recognition of emotional similarity between facial expressions following bilateral amygdala damage. Neuropsychologia, 37, 1135-1141.

Hamann, S. B., Stefanacci, L., Squire, L. R., Adolphs, R., Tranel, D., Damasio, H., et al. 1996. Recognizing facial emotion. Nature, 379, 497.

Hurd, Y. L., McGregor, A., \& Ponten, M. 1997. In vivo amygdala dopamine levels modulate cocaine selfadministration behaviour in the rat: D1 dopamine receptor involvement. European Journal of Neuroscience, 9, 2541-2548.

Joseph, M. H., Datla, K., \& Young, A. M. 2003. The interpretation of the measurement of nucleus accumbens dopamine by in vivo dialysis: the kick, the craving or the cognition? Neuroscience and Biobehavioral Reviews, 27, 527-541.

Kahneman, D. 2002. Maps of bounded rationality: A perspective on intuitive judgment and choice. In T. Frangsmyr (Ed.), Les Prix Nobel (pp. 416-499). Akademibokhandeln City: Almqvist \& Wiksell International.

Karnath, H. O. 2001. New insights into the functions of the superior temporal cortex. Nature Reviews Neuroscience, 2, 568-576.

Kim, H., Somerville, L. H., Johnstone, T., Alexander, A. L., \& Whalen, P. J. 2003. Inverse amygdala and medial prefrontal cortex responses to surprised faces. Neuroreport, 14, 2317-2322.

Kling, A. S., \& Brothers, L. A. 1992. The amygdala and social behavior. In J. P. Aggleton (Ed.), The amygdala: Neurobiological aspects of emotion, memory, and mental dysfunction (pp. 353-377). New York: Wiley-Liss.

Kubota, Y., Sato, W., Murai, T., Toichi, M., Ikeda, A., \& Sengoku, A. 2000. Emotional cognition without awareness after unilateral temporal lobectomy in humans. The Journal of Neuroscience, 20, RC97: 15.

LeDoux, J. E. 1996. The emotional brain: The mysterious underpinnings of emotional life. New York: Simon and Schuster.

Louilot, A., Simon, H., Taghzouti, K., \& Le Moal, M. 1985. Modulation of dopaminergic activity in the nucleus accumbens following facilitation or blockade of the dopaminergic transmission in the amygdala: a study by in vivo differential pulse voltammetry. Brain Research, 346, 141-145.

Maeda, H., Morimoto, H., \& Yanagimoto, K. 1993. Response characteristics of amygdaloid neurons provoked by emotionally significant environmental stimuli in cats, with special reference to response durations. Canadian Journal of Physiology and Pharmacology, 71, 374-378.

Niedenthal, P. M., Halberstadt, J. B., \& Innes-Ker, A. H. 1999. Emotional Response Categorization. Psychological Review, 106, 337-361.

Ono, T., \& Nishijo, H. 1999. Neurophysiological basis of emotion in primates: Neuronal responses in the monkey amygdala and anterior cingulate cortex. In S. M. Gazzaniga (Ed.), The new cognitive neurosciences (2nd ed., pp. 1099-1114). Cambridge: MIT Press.

Perrett, D. I. 1999. A cellular basis for reding minds from faces and actions. In M. D. Hauser, \& M. Konishi (Eds.), The design of animal communication (pp. 159-185). Cambridge: MIT Press.

Philippot, P. 1993. Inducing and assessing differentiated emotion-feeling states in the laboratory. Cognition and Emotion, 7, 171-193.

Phillips, M. L., Bullmore, E. T., Howard, R., Woodruff, P. W., Wright, I. C., Williams, S. C., et al. 1998. Investigation of facial recognition memory and happy and sad facial expression perception: an fMRI study. Psychiatry Research, 83, 127-138.

Phillips, M. L., Young, A. W., Scott, S. K., Calder, A. J., Andrew, C., Giampietro, V., et al. 1998. Neural responses to facial and vocal expressions of fear and disgust. Proceedings of the Royal Society of London Series B: Biological Sciences, 265, 1809-1817.

Rapcsak, S. Z., Galper, S. R., Comer, J. F., Reminger, S. L., Nielsen, L., Kaszniak, A. W., et al. 2000. Fear recognition deficits after focal brain damage: A cautionary note. Neurology, 54, 575-581.

Russchen, F. T., Bakst, I., Amaral, D. G., \& Price, J. L. 1985. The amygdalostriatal projections in the monkey. An anterograde tracing study. Brain Research, 329, 241-257.

Sato, W., Kubota, Y., Okada, T., Murai, T., Yoshikawa, S., \& Sengoku, A. 2002. Seeing happy emotion in fearful and angry faces: Qualitative analysis of the facial expression recognition in a bilateral amygdala damaged patient. Cortex, 38, 727-742.

Sato, W., Yoshikawa, S., Kochiyama, T., \& Matsumura, M. 2004. The amygdala processes the emotional 
significance of facial expressions: An fMRI investigation using the interaction between expression and face direction. Neuroimage, 22, 1006-1013.

Schmolck, H., \& Squire, L. R. 2001. Impaired perception of facial emotions following bilateral damage to the anterior temporal lobe. Neuropsychology, 15, 30-38.

Scott, S. K., Young, A. W., Calder, A. J., Hellawell, D. J., Aggleton, J. P., \& Johnson, M. 1997. Impaired auditory recognition of fear and anger following bilateral amygdala lesions. Nature, 385, 254-257.

Simon, H., Taghzouti, K., Gozlan, H., Studler, J. M., Louilot, A., Herve, D., et al. 1988. Lesion of dopaminergic terminals in the amygdala produces enhanced locomotor response to D-amphetamine and opposite changes in dopaminergic activity in prefrontal cortex and nucleus accumbens. Brain Research, 447, 335-340.

Sprengelmeyer, R., Young, A. W., Schroeder, U., Grossenbacher, P. G., Federlein, J., Buttner, T., et al. 1999. Knowing no fear. Proceedings of the Royal Society of London Series B: Biological Sciences, 266, 2451-2456.

Stefanacci, L., \& Amaral, D. G. 2000. Topographic organization of cortical inputs to the lateral nucleus of the macaque monkey amygdala: a retrograde tracing study. Journal of Comparative Neurology, 421, 52-79.

Stevenson, C. W., \& Gratton, A. 2003. Basolateral amygdala modulation of the nucleus accumbens dopamine response to stress: role of the medial prefrontal cortex. European Journal of Neuroscience, 17, 1287-1295.

Stevenson, C. W., \& Gratton, A. (in press). Role of basolateral amygdala dopamine in modulating prepulse inhibition and latent inhibition in the rat. Psychopharmacology.

Stevenson, C. W., Sullivan, R. M., \& Gratton, A. 2003. Effects of basolateral amygdala dopamine depletion on the nucleus accumbens and medial prefrontal cortical dopamine responses to stress. Neuroscience, 116, 285-293.

Stone, V. E., Baron-Cohen, S., Calder, A., Keane, J., \& Young, A. 2003. Acquired theory of mind impairments in individuals with bilateral amygdala lesions. Neuropsychologia, 41, 209-220.

Swanson, L. W., \& Petrovich, G. D. 1998. What is the amygdala? Trends in Neurosciences, 21, 323-331.

Vargas, M. F. 1986. Louder than words: An introduction to nonverbal communication. Ames: Iowa State University Press.

Whalen, P. J., Rauch, S. L., Etcoff, N. L., McInerney, S. C., Lee, M. B., \& Jenike, M. A. 1998. Masked presentations of emotional facial expressions modulate amygdala activity without explicit knowledge. The Journal of Neuroscience, 18, 411-418.

Young, A. W., Aggleton, J. P., Hellawell, D. J., Johnson, M., Broks, P., \& Hanley, J. R. 1995. Face processing impairments after amygdalotomy. Brain, 118, 15-24.

Young, A. W., Hellawell, D. J., van de Wal, C., \& Johnson, M. 1996. Facial expression processing after amygdalotomy. Neuropsychologia, 34, 31-39.

(Manuscript received May 28, 2004; Revision accepted June 25, 2004) 